\title{
Ações Judiciais e Demandas Administrativas na Garantia do Direito de Acesso a Medicamentos EM FLORIANÓPOLIS-SC ${ }^{(*)}$
}

\author{
LEGALACTIONS AND ADMINISTRATIVE DEMANDS RELATED TO THE \\ GUARANTEE OF THE RIGHTTO DRUGS ACCESS IN THE \\ CITY OF FLORIANÓPOLIS-SC
}

\author{
Silvana Nair Leite ${ }^{(* *)}$ \\ Sônia Maria Polidório Pereira ${ }^{(* * *)}$ \\ Patrícia da Silva ${ }^{(* * * *)}$ \\ José Miguel do Nascimento Jr. (*****) $^{(* * * *)}$ \\ Benedito Carlos Cordeiro ${ }^{(* * * * * * *)}$ \\ Ana Paula Veber ${ }^{(* * * * * * *)}$
}

\section{RESUMO}

O estudo objetivou analisar as solicitações de medicamentos por usuários individuais, de 2003 a 2006, no município de Florianópolis. Foram analisadas 2.426 autorizações para fornecimento de 5.645 medicamentos e 5.283 produtos correlatos na Secretaria de Saúde e na Secretaria da Criança, Adolescente, Idoso, Família e Desenvolvimento Social (SMDS) e na Associação Florianopolitana de Voluntários, considerando os tipos de medicamentos solicitados, valores empregados e características dos solicitantes. A Secretaria de Saúde recebeu, em comparação à SMDS e à Associação Florianopolitana de Voluntários, o maior número de solicitações em todos os anos e as autorizações de compra não apresentavam todos os dados sobre as so-

(*) Apresentado parcialmente no IV Congresso Brasileiro de Ciências Sociais e Humanas em Saúde - Abrasco, Salvador, julho de 2007.

${ }^{* *}$ ) Doutora em Saúde Pública pela Universidade de São Paulo (USP), professora do Programa de Mestrado em Saúde e Gestão do Trabalho da Universidade do Vale do Itajaí (UNIVALI), consultora do Departamento de Assistência Farmacêutica/SCTIES/Ministério da Saúde. E-mail: snleite @ univali.br. $\left.{ }^{* \star *}\right)$ Mestre em Saúde e Gestão do Trabalho.

$\left(^{* * * *}\right)$ Acadêmica de Farmácia da UNIVALI.

$\left.{ }^{* * * * *}\right)$ Mestre em Saúde Pública pela Universidade Federal de Santa Catarina (UFSC).

$\left(^{* * * * * *)}\right.$ Doutor em Saúde Pública pela USP, professor do Curso de Farmácia da UNIVALI.

$\left({ }^{\star \star \star \star \star \star \star}\right)$ Mestre em Farmácia pela UFSC.

Recebido em 30.05.08. Revisado em 20.10.08. Aprovado em 03.11.08. 
licitações, sendo que, o principal tipo de demanda atendido foi via decisão judicial. Na SMDS, a principal demanda era interna. A classe de medicamentos mais solicitada, via mandado judicial ou não, foi a dos que atuam no Sistema Nervoso, sendo Carbamazepina o principal medicamento, enquanto o medicamento Pimecrolimo, por via dermatológica, foi o mais solicitado e o que representou maior custo. Os medicamentos estavam prescritos, em sua maioria, pelo nome comercial e $32 \%$ constavam de programas de fornecimento de medicamentos padronizados pelo SUS. Conclui-se que o fornecimento de medicamentos por compra direta não está adequadamente inserido na gestão da assistência farmacêutica municipal, desrespeitando a Política Nacional de Medicamentos.

\title{
Palavras-chave
}

Acesso Universal a Serviços de Saúde; Assistência Farmacêutica; Custos de Medicamentos; Política Nacional de Medicamentos.

\begin{abstract}
This study aims at analyzing the requests for drugs by individual users, from 2003 to 2006, in the city of Florianópolis. It was analyzed 2,426 authorizations for the supply of 5,645 drugs and 5,283 correlated products in the Secretary for Health, the Secretary for Social Development and in the Volunteering Association of Florianópolis, considering the types of drugs requested, costs and characteristics of those who requested them. Secretary of Health received the highest number of requests throughout the years and the authorizations for purchase did not present all the data of the requests. The main type of demand met by the Secretary of Health was originated from judicial decisions, while the main type met by the Secretary for Social Development was internal demands. The most frequently requested class of drugs, whether by legal writ or not, was the one which affects the Nervous System, with Carbamazepine being the primary medication, while a Pimecrolimus cream was the most frequently requested medication representing the highest cost. The majority of the drugs were prescribed by their commercial name and $32 \%$ of them were standardized by the Brazilian National Health System. It is concluded that the supply of drugs by direct purchase is not adequately dealt by the municipal pharmaceutical assistance, disrespecting the National Drug Policy.
\end{abstract}

\section{Keywords}

Brazilian National Drug Policy; Drug Costs; Pharmaceutical assistance; Universal Access to Health Services. 


\section{INTRODUÇÃO}

Um dos pilares da atenção à saúde é a assistência farmacêutica, cujo objetivo é disponibilizar e racionalizar o uso de medicamentos para o atendimento às necessidades da população, consolidando o processo de descentralização e trazendo responsabilidades aos gestores das três esferas do governo, em busca da otimização e eficácia da aplicação dos produtos e da alocação dos recursos financeiros ${ }^{(1)}$.

Destaca-se, neste aspecto, a aprovação da Política Nacional de Medicamentos, em 1998, que propõe garantir a segurança, a eficácia e a qualidade dos medicamentos ao menor custo possível e promover o uso racional e o acesso da população às drogas consideradas essenciais. Para isto, estabelece diretrizes e prioridades, observados os princípios constitucionais ${ }^{(2)}$.

Assegurar a equidade e o acesso aos medicamentos garantidos pelo SUS torna-se um desafio da municipalização dos serviços, pois, os medicamentos são considerados insumos fundamentais, utilizados na prevenção, no diagnóstico e tratamento de várias enfermidades, sendo o seu uso a intervenção terapêutica mais frequentemente empregada ${ }^{(3)}$. No entanto, a grande variedade de produtos farmacêuticos disponíveis no mercado farmacêutico, a influência da indústria farmacêutica e a cultura difundida entre usuários de utilização de medicamentos constituem pressões para a necessidade de prescrição medicamentosa. Muitas vezes, os profissionais prescritores têm acesso limitado à informação objetiva sobre os fármacos que prescrevem e dispensam; resultado da falta de hábito de busca, mais do que indisponibilidade de informação(4). Como consequência, observa-se o uso irracional de medicamentos, o que colabora para os problemas de acesso a estes produtos.

Acesso aos medicamentos, para Luiza e Bermudez ${ }^{(5)}$, envolve uma complexa rede de atores e instituições públicas e privadas que intervêm no processo de atenção à saúde. No Brasil, especificamente, a questão do acesso aos medicamentos é marcada pela garantia constitucional do direito social à saúde.

(1) BRASIL. Ministério da Saúde. Secretaria de Ciências, Tecnologia e Insumos Estratégicos. Departamento de Assistência Farmacêutica e Insumos Estratégicos. Assistência farmacêutica na atenção básica: instruções técnicas para sua organização. Brasília: Ministério Público, 2006.

(2) Ministério da Saúde. Portaria GM n. 3.916, 30 de outubro de 1998. Aprova a Política Nacional de Medicamentos. Diário Oficial [da] República Federativa do Brasil, Poder Executivo, Brasília, DF, 10 nov. 1998b. s. 1, n. 215-E, p. 18.

(3) LUIZA, V. L.; BERMUDEZ, J. A. Z. Acesso a medicamentos: conceitos e polêmicas. In: BERMUDEZ, J. A. Z.; OLIVEIRA, M. A. Esher A. Acesso a medicamentos: derecho fundamental. Papel del Estado. Rio de Janeiro: ENSP, 2004. p. 45-67.

(4) WANNMACHER, L. Uso racional de medicamentos: medida facilitadora do acesso. In: BERMUDEZ, J. A. Z.; OLIVEIRA, M. A. Esher A. op. cit., p. 91-101.

(5) LUIZA, V. L.; BERMUDEZ, J. A. Z. op. cit., p. 45-67. 
Entre as estratégias aplicadas para a garantia deste direito, um fenômeno vem crescendo recentemente e tem sido considerado grande ameaça à sanidade financeira e administrativa do Sistema Único de Saúde (SUS): os mandados judiciais para fornecimento de medicamentos. Messeder et al(6) descrevem o acesso a medicamentos no Estado do Rio de Janeiro por este meio, revelando o fornecimento de medicamentos essenciais e de inovações tecnológicas, muitos dos quais prescritos sem o cumprimento das diretrizes e protocolos terapêuticos já estabelecidos. Vieira e Zucchi(7) também descrevem esta situação na cidade de São Paulo, destacando a falta de observância das diretrizes do SUS nesta forma de fornecimento de medicamentos.

Tal fenômeno vem completar outras práticas já arraigadas nos serviços públicos: o pedido individual por produtos ou serviços diretamente aos gestores do sistema público e o fornecimento de medicamentos pelos serviços administrativos das secretarias da saúde e de assistência social dos municípios. A manutenção deste serviço pela assistência social, mesmo após a implantação do SUS, possivelmente deve-se à cultura oriunda do processo histórico de construção das políticas de saúde no Brasil, intimamente relacionado às políticas de assistência social, como a previdenciária. Apesar de ser fato comum, não se encontra literatura ou discussão sobre este tema.

O fornecimento de medicamentos por estas vias é possibilitado pela compra direta por estas instituições. O objetivo desta pesquisa foi analisar o fornecimento de medicamentos por compra direta, via judicial ou não, pela Secretaria Municipal de Saúde (SMS), Secretaria Municipal da Criança, Adolescente, Idoso, Família e Desenvolvimento Social (SCAIFDS) e a Associação Florianopolitana de Voluntários (AFLOV) do município de Florianópolis (SC) entre os anos de 2003 e 2006. Conhecer esta prática é essencial para avaliar a forma de acesso aos medicamentos por usuários em uma cidade, além de indicar o grau de organização gerencial e política da assistência farmacêutica no nível municipal e sua relação com as políticas de assistência social.

\section{MATERIAIS E MÉTODOS}

\section{Local do Estudo}

Florianópolis, Capital do Estado de Santa Catarina, atualmente possui 396.778 habitantes, com população predominante urbana com taxa média

(6) MESSEDER N. M.; CASTRO DE, C. G. S. O.; LUIZA, V. L. Mandados judiciais como ferramentas para garantia do acesso a medicamentos no setor público: a experiência do Estado do Rio de Janeiro. Cadernos de Saúde Pública, São Paulo, v. 21, n. 2, p. 525-534, 2005.

(7) VIEIRA, F. S.; ZUCCHI, P. Distorções causadas pelas ações judiciais à política de medicamentos no Brasil. Revista de Saúde Pública, São Paulo, v. 41, n. 2, p. 214-222, 2007. 
de crescimento anual de $3 \%$, ocupando uma área de $435,79 \mathrm{~km}^{2}$. A Secretaria Municipal de Saúde (SMS), a Secretaria Municipal da Criança, Adolescente, Idoso, Família e Desenvolvimento Social (SCAIFDS) do município de Florianópolis e a Associação Florianopolitana de Voluntários (AFLOV) compõem os órgãos onde foram resgatados os dados, utilizando respectivamente seus departamentos administrativo e financeiro.

\section{Coleta e análise de dados}

Os dados foram obtidos dos arquivos de cadastros de autorizações financeiras do universo das autorizações de compras disponíveis dos anos de 2003 a 2006 da SMS, da SCAIFDS e da AFLOV, do município de Florianópolis. Todas as autorizações de compras de medicamentos para atender solicitações de pacientes individuais, independentemente da origem — judicial ou administrativa, foram identificadas nos arquivos. Nestas autorizações foram coletados os seguintes dados: os medicamentos pleiteados; produtos correlatos solicitados, apenas quando na mesma solicitação de um ou mais medicamentos; identificação dos medicamentos constantes na Relação Municipal de Medicamentos Essenciais ou de Programas Estratégicos; recursos do município gastos no atendimento das solicitações; serviço de origem do usuário; data da solicitação; forma de encaminhamento para a solicitação. Todos os medicamentos encontrados foram classificados com base no "Código Anatômico, Terapêutico e Químico" — código ATC. Os dados constituíram um banco de dados no programa Excel e foram analisados por médias e porcentagens. O projeto de pesquisa foi aprovado pela Comissão de Ética em Pesquisa da Universidade do Vale do Itajaí (UNIVALI) (Parecer n. 175/06).

\section{RESULTADOS}

Foram encontradas 2.426 autorizações para fornecimento de 5.645 medicamentos e 5.283 produtos correlatos, entre 2003 e 2006. A SMS recebeu o maior número de solicitações em todos os anos. Na SCAIFDS, só foi possível identificar autorizações a partir de 2005 e na AFLOV, apenas de 2006. No ano de 2005, a SCAIFDS passou a fornecer parte dos medicamentos e em 2006, esta atividade foi dividida também com a AFLOV, a pedido da administração municipal (Tabela 1). O número de usuários atendidos por esta via subiu de 5 para 89 entre 2003 e 2004, para 220 em 2005 e 298 em 2006. 
Tabela 1. Número de solicitações, usuários, medicamentos, produtos correlatos e valores empregados pelas instituições, agrupado por ano de solicitação e instituição fornecedora

\begin{tabular}{|c|c|c|c|c|c|c|c|c|c|c|c|c|c|c|c|}
\hline \multirow[t]{2}{*}{ Ano } & \multicolumn{3}{|c|}{ N. Solicitações } & \multicolumn{3}{|c|}{ N. Usuários } & \multicolumn{3}{|c|}{ N. Medicamentos } & \multirow{2}{*}{\multicolumn{3}{|c|}{$\begin{array}{c}\text { N. Outros } \\
\text { SMS SMDS AFLOV }\end{array}$}} & \multicolumn{2}{|c|}{ Valores R\$ } & \multirow[b]{2}{*}{ AFLOV } \\
\hline & SMS & SMDS & AFLOV & SMS & SMDS & AFLOV & SMS & SMD & AFLOV & & & & SMS & SMDS & \\
\hline 2003 & 22 & - & - & 5 & - & - & 100 & - & - & 232 & - & - & $3.398,96$ & - & - \\
\hline 2004 & 298 & - & - & 89 & - & - & 933 & - & - & 759 & - & - & $30.695,08$ & - & - \\
\hline 2005 & 932 & 123 & - & 155 & 65 & - & 2.114 & 159 & - & 2.381 & 22 & - & $157.828,87$ & $13.796,45$ & - \\
\hline 2006 & 695 & 156 & 200 & 126 & 89 & 83 & 1.727 & 211 & 401 & 1.831 & 49 & 9 & $137.429,05$ & $14.834,27$ & $16.676,53$ \\
\hline Total & 1.947 & 279 & 200 & 375 & 154 & 83 & 4.874 & 370 & 401 & 5.203 & 71 & 9 & $329.351,96$ & $28.630,72$ & $16.676,53$ \\
\hline
\end{tabular}

O valor total empregado pela Prefeitura Municipal foi de $\mathrm{R} \$ 374.659,21$ (valor não corrigido). Do ano de 2003 para o ano de 2004, na SMS, houve um aumento de 9 vezes no valor empregado com esta forma de fornecimento de medicamentos, mantendo a tendência de crescimento nos anos seguintes. Em um caso observado, uma única usuária recebeu medicamentos entre 2004 e 2006 no valor aproximado de $R \$ 35.000,00$. O valor empregado apenas com atendimento de demandas do poder judiciário em 2006, adquiridos pela SMS por compra direta, foi de $\mathrm{R} \$ 95.211,75$.

As autorizações da SMS apresentaram o menor número de informações a respeito do usuário solicitante (como endereço, telefone, origem da prescrição, diagnóstico, prescritor). Em média, $20 \%$ das autorizações continham todas estas informações. Para SCAIFDS e AFLOV, os serviços que mais originaram prescrições atendidas nestas instituições foram os públicos não hospitalares (unidades básicas e policlínicas). Entre os diagnósticos relatados nas autorizações, os mais frequentemente citados foram Diabetes Mellitus na SMS e SCAIFDS, Hipertensão Arterial Sistêmica e neoplasias na AFLOV.

A Tabela 2 demonstra os tipos de encaminhamento das solicitações para o fornecimento de medicamentos por compra direta. Na SMS, $47 \%$ das autorizações foram resultantes de decisões judiciais, fenômeno observado apenas a partir de 2004 e com grande crescimento nos anos seguintes, chegando a representar $77,23 \%$ das demandas nesta via de acesso em 2006 (as demandas que constam como administrativas internas neste ano são resultantes de acordo com o Ministério Público, não sendo computadas como mandados judiciais); em $22,8 \%$ das autorizações não foi possível verificar o tipo de encaminhamento. Como demanda administrativa interna foram classificados os encaminhamentos de departamentos e autorizações do Gabinete (Prefeitura ou Secretaria de Saúde), correspondendo a 17,21\%; como demanda administrativa externa foram classificados os encaminhamentos por políticos da cidade, demanda espontânea e de outras Secretarias, forma de encaminhamento que não foi registrada no último ano. O Ministério Público foi citado em pequena parcela das autorizações como determinante do fornecimento. Na SCAIFDS e AFLOV, 33,4\% não continham esta informação e a demanda administrativa interna foi a principal forma de encaminhamento, com $45,72 \%$ das autorizações. 
Tabela 2. Secretaria da Saúde: classificação pelo tipo de encaminhamento de cada solicitação

\begin{tabular}{|ccccc|}
\hline Ano & $\begin{array}{c}\text { Decisão } \\
\text { Judicial }\end{array}$ & $\begin{array}{c}\text { Demanda } \\
\text { Administrativa } \\
\text { Interna }\end{array}$ & $\begin{array}{c}\text { Ministério } \\
\text { Público }\end{array}$ & $\begin{array}{c}\text { Não possui } \\
\text { informação }\end{array}$ \\
\hline \multirow{2}{*}{$\mathbf{2 0 0 3}$} & 0 & 8 & 0 & 13 \\
2004 & $(0,00 \%)$ & $(36,36 \%)$ & $(0,00 \%)$ & $(59,09 \%)$ \\
& $(15,10 \%)$ & $(9,73 \%)$ & $(4,36 \%)$ & $(49,33 \%)$ \\
2005 & $(36,48 \%)$ & $(15,56 \%)$ & $(4,51 \%)$ & $(26,18 \%)$ \\
& 502 & 153 & 0 & 40 \\
2006 & $(72,23 \%)$ & $(22,01 \%)$ & $(0,00 \%)$ & $(5,76 \%)$ \\
& 915 & 335 & 55 & 444 \\
Total & $(47,00 \%)$ & $(17,21 \%)$ & $(2,82 \%)$ & $(22,80 \%)$ \\
\hline
\end{tabular}

Os medicamentos fornecidos por compra direta eram, na sua maioria, prescritos por seus nomes comerciais: $76,63 \%$, ou 4.326 medicamentos. Medicamentos constantes da Relação Municipal de Medicamentos Essencias (REMUME) do município representaram $21,15 \%$ do total solicitado; constantes do Programa de Medicamentos Excepcionais, 9,07\% e medicamentos fornecidos pelo Programa de Saúde Mental, 12,01\% (Tabela 3).

Tabela 3. Medicamentos classificados por tipo de financiamento para aquisição e por ano de solicitação

\begin{tabular}{|cccccc|}
\hline Ano & RESUME & $\begin{array}{c}\text { Medicamentos } \\
\text { Excepcionais }\end{array}$ & $\begin{array}{c}\text { Medicamentos } \\
\text { Saúde Mental }\end{array}$ & $\begin{array}{c}\text { Prescrito } \\
\text { pela DCB }\end{array}$ & $\begin{array}{r}\text { Total de } \\
\text { Medicamentos }\end{array}$ \\
\hline 2003 & $(46,00 \%)$ & $(12,00 \%)$ & $(0,00 \%)$ & $(4,00 \%)$ & 100 \\
& 261 & 105 & 186 & 172 & 933 \\
2004 & $(27,97 \%)$ & $(11,25 \%)$ & $(4,36 \%)$ & $(18,44 \%)$ & \\
& 327 & 227 & 289 & 587 & 2.273 \\
2005 & $(14,39 \%)$ & $(9,99 \%)$ & $(4,51 \%)$ & $(25,82 \%)$ & \\
& 560 & 168 & 185 & 556 & 2.339 \\
2006 & $(23,94 \%)$ & $(7,18 \%)$ & $(0,00 \%)$ & $(23,77 \%)$ & \\
& 1.194 & 512 & 678 & 1.319 & 5.645 \\
Total & $(21,15 \%)$ & $(9,07 \%)$ & $(2,82 \%)$ & $(23,37 \%)$ & \\
\hline
\end{tabular}

Fonte: autorizações de compras da SMS de 2003 a 2006, SCAIFDS 2005 a 2006 e AFLOV 2006, Florianópolis, SC. 
A mesma tendência pode ser observada entre os medicamentos demandados por decisão judicial: $32,2 \%$ dos medicamentos solicitados eram padronizados pelo SUS (Tabela 4).

Tabela 4. Medicamentos requeridos por Mandados Judiciais, classificados por tipo de financiamento para aquisição e por ano de solicitação

\begin{tabular}{|cccccc|}
\hline Ano & RESUME & $\begin{array}{c}\text { Medicamentos } \\
\text { Excepcionais }\end{array}$ & $\begin{array}{c}\text { Medicamentos } \\
\text { Saúde Mental }\end{array}$ & $\begin{array}{c}\text { Prescrito } \\
\text { pela DCB }\end{array}$ & $\begin{array}{c}\text { Total de } \\
\text { Medicamentos }\end{array}$ \\
\hline \multirow{2}{*}{2004} & 17 & 31 & 0 & 33 & 136 \\
& $(12,50 \%)$ & $(12,00 \%)$ & $(0,00 \%)$ & $(4,00 \%)$ & \\
2005 & $(11,60 \%)$ & $(11,25 \%)$ & $(4,36 \%)$ & $(18,44 \%)$ & 845 \\
2006 & 299 & 78 & 116 & 323 & 1.291 \\
\multirow{2}{*}{ Total } & $(14,39 \%)$ & $(9,99 \%)$ & $(4,51 \%)$ & $(25,82 \%)$ & \\
& $(18,22 \%)$ & $(7,18 \%)$ & $(0,00 \%)$ & $(23,77 \%)$ & \\
\hline
\end{tabular}

Fonte: autorizações de compras da SMS de 2003 a 2006, Florianópolis, SC.

A classificação pelo código ATC $^{(8)}$ demonstrou que a classe mais solicitada de medicamentos é a dos medicamentos que atuam no Sistema Nervoso $(40,99 \%)$, em todas as instituições fornecedoras, seguida pelos que atuam no Trato Alimentar ou Metabolismo e Sistema Hematopoiético (Figura 1). Entre os medicamentos demandados por decisão judicial, aqueles que atuam no Sistema Nervoso também representam 40\%, sendo a Carbamazepina o medicamento mais solicitado deste grupo; os medicamentos para uso Dermatológico representam $17 \%$ da demanda, com Pimecrolimo como medicamento mais solicitado entre todas as classes terapêuticas, em número de produtos e em valor monetário empregado; os medicamentos que atuam no Trato Alimentar ou Metabolismo correspondem a 14,39\% das demandas judiciais, especialmente pelo fornecimento de Insulinas.

(8) WHO COLLABORATING Centre for Drugs Statistics Methodology. Anatomical Therapeutic Chemical Classification (ATC code). Disponível em: <http://www.whocc.no/ atcddd>. Acesso em: 15 mar. 2007. 
Figura 1. Classificação ATC dos medicamentos solicitados

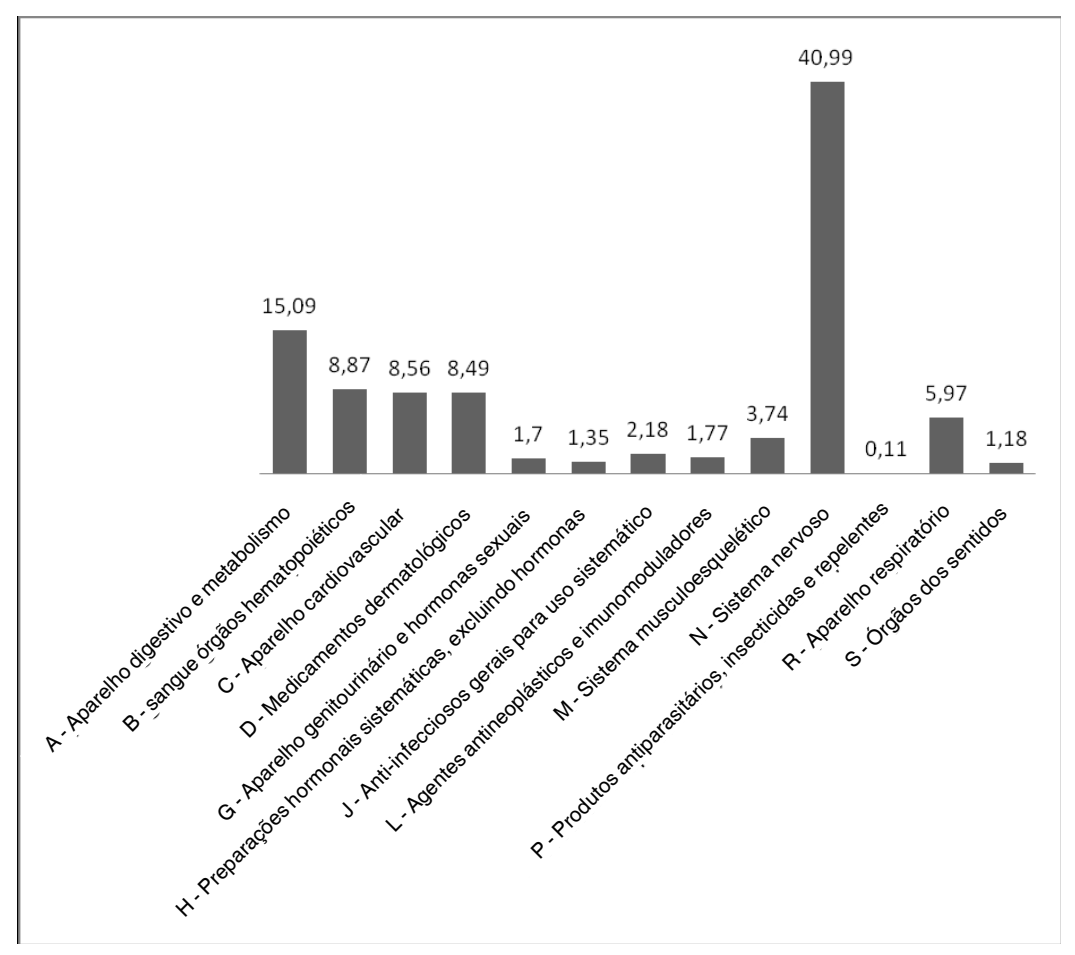

III. DISCUSSÃO

Alguns objetivos iniciais deste estudo não puderam ser concluídos pela limitação de dados disponíveis nas autorizações de compra direta de medicamentos. A falta de informações, principalmente naquelas arquivadas na SMS, comprometeu a análise dos valores empregados no fornecimento de medicamentos, a localização geográfica dos usuários, a caracterização geral dos diagnósticos, as especialidades dos prescritores e o tempo de espera para o fornecimento dos medicamentos solicitados. A impossibilidade de determinar as condições sociais de cada usuário solicitante inviabilizou também a discussão da equidade no acesso aos medicamentos, fato também observado no estudo de Vieira e Zucchi(i). A falta de informações nas autorizações revela que o processo para o fornecimento de medicamentos

(9) VIEIRA, F. S.; ZUCCHI, P. op. cit. 
não recebe a atenção devida quanto a seus aspectos burocráticos, inclusive com respeito aos critérios para o recebimento de um benefício da assistência social (no caso das solicitações à SCAIFDS e AFLOV), como a renda. A avaliação da racionalidade da aplicação destes medicamentos também é impossibilitada pelo conteúdo insuficiente de informações sobre diagnósticos e tratamentos anteriores.

O número de usuários e solicitações na SMS diminuiu no último ano devido ao atendimento de parte da demanda pelas outras instituições. Segundo informações fornecidas pela SMS, a partir de 2006 esta secretaria passou a priorizar o atendimento de demandas judiciais e solicitou à SCAIFDS que atendesse às demais. A AFLOV, que é uma organização não governamental que presta serviços para a Prefeitura Municipal, por sua vez, passou a absorver parte da demanda da SCAIFDS em 2006.

O valor empregado pela administração municipal com esta forma de fornecimento em termos absolutos $(R \$ 95.211,75)$ pode não ser significativo em relação ao montante do investimento na área; porém, corresponde, para o ano de 2006 , a $R \$ 566,90$ por usuário solicitante, enquanto o total empregado na compra de medicamentos padronizados para a atenção básica no município correspondeu a $\mathrm{R} \$ 17,32$ por habitante (segundo informado pela SMS).

O fenômeno da intervenção do poder judiciário na garantia do direito social

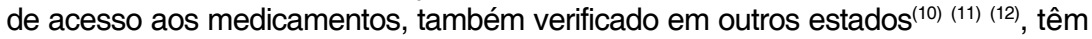
sido alvo de preocupação entre gestores e pesquisadores nas áreas de saúde pública e direito.

Em que pese o direito constitucional e infraconstitucional que garante o direito de acesso aos medicamentos, a proteção judicial a este direito vem sendo criticada pelos que entendem que o atendimento às demandas individuais do direito à saúde prejudica o atendimento coletivo ${ }^{(13)}$. Nesta mesma linha de raciocínio, Marques ${ }^{(14)}$ alerta que o Poder Judiciário, ao garantir o direito de acesso aos medicamentos pela via judicial, não considera a dimensão política de garantir este mesmo direito para toda a coletividade, ou seja, estaria acontecendo uma "judicialização" da política de assistência farmacêutica, com o Poder Judiciário exercendo atitudes do Poder Executivo como, por exemplo, a decisão alocativa de recursos em saúde.

Também Costa(15), ao comentar a decisão proferida em 2004 de Suspensão de Tutela Antecipada STA 59/SC, do Superior Tribunal de Justiça

(10) BRASIL. Ministério da Saúde. op. cit.

(11) MESSEDER N. M.; CASTRO DE, C. G. S. O.; LUIZA, V. L. op. cit., p. 525-534.

(12) VIEIRA, F. S.; ZUCCHI, P. op. cit.

(13) DALLARI, D. A. Regulação de medicamentos. Revista de Direito Sanitário, São Paulo, v. 7, n. 1-3, p. 111-116, 2006.

(14) MARQUES, S. B. A garantia do direito social à assistência farmacêutica: o papel do sistema jurídico e do sistema político. Revista de Direito Sanitário, São Paulo, v. 7, n. 1-3, p. 195-217, 2006. (15) COSTA, L. F. G. Listagem de medicamentos e a decisão proferida na STA n. 59-STJ: a justiciabilidade dos direitos sociais. Revista de Direito Sanitário, São Paulo, v. 5, n. 1, p. 119-135, 2004. 
(STJ), recomenda o exercício harmonioso dos três poderes da República, pois, a implementação de políticas públicas deve compatibilizar a efetivação dos direitos sociais com as limitações materiais do Estado.

Vale a pena comentar ainda as decisões recentes do Supremo Tribunal Federal (STF) em 2007, proferidas pela então Presidente, Ministra Ellen Gracie. Nos processos de Suspensão de Tutela Antecipada STA n. 91/AL e Suspensão da Segurança SS n. 3073/RN, a Ministra reconhece os argumentos expostos acima, ou seja, atender a toda e qualquer demanda judicial individual de medicamentos, como direito público subjetivo, diminui "a possibilidade de oferecimento de serviços básicos ao restante da coletividade". Por outro lado, ao emitir as decisões referentes à Suspensão da Segurança SS n. 3158/RN e SS n. 3205/AM, a Ministra afirma que "a discussão sobre a competência para a execução de programas de saúde e de distribuição de medicamentos não pode se sobrepor ao direito à saúde", indeferindo o pedido de suspensão da entrega de medicamentos, não podendo todos os pedidos ser analisados de forma abstrata e genérica, mas sim analisados caso a caso, de forma concreta $^{(16)}$. Se para alguns estas decisões podem parecer um contrassenso, elas aparentam muito mais a abertura de precedentes (jurisprudência) para que o Estado cumpra o seu papel, ou seja: possibilitar o acesso aos medicamentos para as pessoas que necessitam destes insumos, ao mesmo tempo em que abre a possibilidade de discussão do que pode ser negado pelo Estado em benefício da Sociedade como um todo. Cada vez que o Estado for negligente frente a necessidades que devam ser cumpridas, compete ao Poder Judiciário a garantia deste direito; porém, como bem sinaliza Marques ${ }^{(17)}$ :

como o direito à assistência farmacêutica depende de uma política pública e de recursos públicos para ser garantido, sob a perspectiva da justiça distributiva, é preciso que essas necessidades individuais sejam contextualizadas dentro da política pública de medicamentos. Assim, a noção de justiça distributiva pode nortear a prestação coletiva e o próprio atendimento às necessidades terapêuticas individuais.

Além da forma judicial de encaminhamento para o acesso, as demandas internas e externas e o fornecimento de medicamentos pela SCAIFDS e uma organização da área social, como a AFLOV, merecem atenção. As demandas internas e externas sugerem o estabelecimento de formas de acesso a medicamentos por favorecimento pessoal: conhecidos que trabalham na gestão, interferência de políticos, entre outras possibilidades. Da mesma forma que vem ocorrendo com os mandados judiciais, em que os impetrantes não correspondem à parcela da população com maior grau de exclusão social(18), estes encaminhamentos não favorecem necessariamente a popu-

(16) SUPREMO TRIBUNAL FEDERAL — STF. Disponível em: <http://www.stf.gov.br> .

(17) MARQUES, S. B. A. op. cit., p. 195-217.

(18) VIEIRA, F. S.; ZUCCHI, P. op. cit. 

Nascimento Jr./Benedito Carlos Cordeiro/ Ana Paula Veber

lação com maiores necessidades financeiras e sim aqueles que têm melhores oportunidades de reivindicar ou negociar seus direitos. Utilizando o estudo de Travassos et a/(19) para esta discussão, é importante ressaltar que os autores relatam que as pessoas mais necessitadas têm menor chance de consumir serviços de saúde, inclusive medicamentos, no Brasil. Além disso, estudo realizado em Santa Catarina revela que a concretização do direito de acesso à saúde pelo acesso a medicamentos via judicial não está promovendo, necessariamente, a conscientização cidadã dos beneficiários, que entendem este processo como um favor de alguém (o médico, o advogado, o político) que o ajudou a conseguir o medicamento que, não legítimo, pode ser retirado a qualquer momento(20).

Já o fornecimento de medicamentos via SCAIFDS e AFLOV tem como prioridade o atendimento de demandas de famílias caracterizadas como de baixa renda e com necessidades sociais. No entanto, estas instituições não estão legal e tecnicamente habilitadas para a atividade de dispensação de medicamentos. Qualquer possibilidade de orientação para o uso correto dos medicamentos ou de avaliação da racionalidade do uso é inviabilizada neste âmbito.

O serviço de origem das prescrições era pouco relatado nas autorizações da SMS. Na SCAIFDS e AFLOV, pode ser observado que a maioria das prescrições era oriunda de serviços ambulatoriais do SUS. A prescrição dos medicamentos pela denominação genérica, apesar de ter aumentado de 2003 para 2006, ainda é muito baixa. No âmbito do SUS, no entanto, a prescrição pela Denominação Comum Brasileira (DCB) é obrigatória. Entre os medicamentos fornecidos por mandados judiciais, da mesma forma os nomes comerciais prevalecem, determinando a compra de medicamentos pelo setor público sem respeito ao menor custo e privilegiando explicitamente as indústrias farmacêuticas detentoras das marcas solicitadas. Um reflexo disto é que, para um mesmo medicamento, foram encontrados valores de $R \$ 24,00$ a $R \$ 250,00$ registrados nas autorizações de compra direta.

O fornecimento de medicamentos constantes da REMUME e do Programa de Saúde Mental do município através de compra direta indica duas possibilidades: houve problemas na organização do serviço de assistência farmacêutica, especialmente no que concerne à programação e aquisição dos medicamentos, ocasionando a falta destes produtos na rede de serviços; ou houve problemas de comunicação entre os setores do poder público, determinando que produtos disponíveis na rede, adquiridos por licitação,

(19) TRAVASSOS, C.; VIACAVA F.; FERNANDES C.; ALMEIDA, C. M. Desigualdades geográficas e sociais na utilização de serviços de saúde no Brasil. Ciência \& Saúde Coletiva, São Paulo, v. 5, n. 1, p. 133-149, 2000.

(20) LEITE, S. N.; MAFRA, A. C. Que direito? Trajetórias e percepções de usuários no processo de acesso a medicamentos por mandados judiciais no estado de Santa Catarina. Ciência \& Saúde Coletiva on-line. Disponível em: <www.cienciaesaudecoletiva.com.br>. 
fossem substituídos pelos mesmos medicamentos comprados sem licitação. Um dos medicamentos mais solicitados neste estudo, a Carbamazepina, consta da REMUME e possui seu fornecimento ao município pelo Programa de Saúde Mental, exemplificando este fato.

Medicamentos constantes do Programa de Medicamentos Excepcionais ou de Alto Custo também foram fornecidos pela Prefeitura Municipal. Neste caso, havia falta dos medicamentos no serviço responsável ou mandados judiciais obrigaram o município a fornecer medicamentos que não são de sua competência, fato também descrito por Vieira e Zucchi(21) no município de São Paulo. Avaliando apenas os medicamentos demandados por via judicial, ocorre a mesma proporção de medicamentos constantes destas listas: $32,2 \%$ são distribuídos pelo SUS. Esta frequência é menor que a relatada pelos estudos de Messeder et al(22) e de Vieira e Zucchi(23).

Assim como verificado no Estado do Rio de Janeiro, a classe de medicamentos mais solicitada por demanda judicial e por solicitação individual em Florianópolis é a dos medicamentos que atuam no Sistema Nervoso. Resultados de estudos de utilização de medicamentos no Brasil mostram uma prevalência de consumo de $9,9 \%$ para os psicofármacos ${ }^{(24)}$ e a crescente demanda populacional por estes medicamentos e suas prescrições é motivo de preocupação. Ao analisar os diagnósticos mais relatados nas autorizações, o Diabetes Mellitus aparece em primeiro lugar na SMS, assim como na cidade de São Paulo(25), porém a grande quantidade de autorizações sem esta informação inviabiliza a comparação com os medicamentos mais solicitados. Na SCAIFDS e AFLOV, Hipertensão Arterial Sistêmica e neoplasias são as patologias mais citadas, mas da mesma forma os medicamentos mais solicitados são os que atuam no Sistema Nervoso.

A Carbamazepina foi o medicamento mais requisitado entre estes. Este medicamento, no entanto, é padronizado e fornecido pelo Programa de Saúde Mental no município.

A segunda classe mais procurada foi a dos medicamentos com ação dermatológica, em razão da grande quantidade de requisições de Pimecrolimo. Este foi o medicamento mais solicitado entre todos e o que representou maior valor monetário empregado. Apesar da restrição da análise ocasionada pela falta de informações relativas ao diagnóstico dos usuários, é importante ressaltar que este medicamento é de segunda linha para suas indicações,

(21) VIEIRA, F. S.; ZUCCHI, P. op. cit.

(22) MESSEDER N. M.; CASTRO DE, C. G. S. O.; LUIZA, V. L.

(23) VIEIRA, F. S.; ZUCCHI, P. op. cit.

(24) RODRIGUES, M. A. P.; FACCHINI, L. A.; LIMA, L. S. Modificações no padrão de consumo de psicofármacos em localidade do sul do Brasil. Revista de Saúde Pública, São Paulo, v. 40, n. 1, p. 107-114, 2006.

(25) VIEIRA, F. S.; ZUCCHI, P. op. cit. 
devendo ser utilizado somente em caso de falha em tratamentos anteriores ${ }^{(26)}$; apresenta ainda um custo muito superior a outros medicamentos com as mesmas indicações. Além disso, há a advertência para ser utilizado pelo menor tempo possível, não existindo até o momento estudos que avaliem sua segurança a longo prazo ${ }^{(27)}$. No presente estudo, um mesmo paciente utilizou o medicamento por 3 anos consecutivamente.

Chama a atenção o fato de que nenhum medicamento antirretroviral foi solicitado. Segundo Messeder et $a^{(28)}$ esta demanda foi mais intensa até 1998 no Estado do Rio de Janeiro e reduzida a partir de então, com a consolidação do Programa Nacional de DST e Aids. A organização local do serviço de atenção ao portador de HIV/Aids, com certeza, também está relacionada com a ausência de solicitações individuais para estes medicamentos junto ao município. No entanto, contra o Estado de Santa Catarina há processos para o fornecimento de medicamentos antirretrovirais.

O Plano Municipal de Assistência Farmacêutica do município reconhece a deficiência de sua organização, pois, este departamento tampouco existe na estrutura organizacional oficial da SMS. Quanto ao fornecimento de medicamentos sob ordem judicial o mesmo plano indica a necessidade de estabelecer diálogo com o Poder Judiciário no sentido de esclarecer as competências por esfera de governo e a necessária racionalização das ações ${ }^{(29)}$. O início deste diálogo ficou formalizado pela Recomendação n. 012/2006/30ª da Promotoria de Justiça da Comarca da Capital ao Secretário Municipal de Saúde de que adotasse providências administrativas imediatas, no âmbito do SUS, no sentido de orientar os profissionais médicos a esgotarem as alternativas de fármacos previstas nos Protocolos Clínicos e Diretrizes Terapêuticas do Ministério da Saúde e demais atos que lhe forem complementares, antes de prescreverem tratamento medicamentoso diverso aos pacientes. Quando da não obediência dos Protocolos, o profissional deverá elaborar fundamentação técnica consistente, e por fim, manifestação sobre possíveis vínculos, formais ou informais, do prescritor com o laboratório fabricante, justificando, assim, a excepcional orientação clínica.

O fornecimento de medicamentos não vinculado a uma organização da assistência farmacêutica municipal, não só pela judicialização, mas também por atendimentos de demandas individuais pela assistência social (inclusive "terceirizado" por uma organização social) compromete a consolidação de propostas como a referida acima. Qualquer proposta de organização e racionalização do acesso e uso de medicamentos no município terá que enfrentar estas formas estabelecidas de acesso, rompendo o ciclo clientelista e fragmentado e investindo em ferramentas de gestão dos serviços de saúde.

(26) VIEIRA, F. S.; ZUCCHI, P. op. cit.

(27) DRUGDEX Drug Evaluations. Pimecrolimus. Thomson Micromedex Healthcare Series, 2007.

(28) MESSEDER, N. M.; CASTRO DE, C. G. S. O.; LUIZA, V. L. op. cit.

(29) FLORIANÓPOLIS. Secretaria Municipal de Saúde. Plano Municipal de Assistência Farmacêutica: 2006-2008. Florianópolis, 2006. 


\section{REFERÊNCIAS BIBLIOGRÁFICAS}

BRASIL. Ministério da Saúde. Secretaria de Ciências, Tecnologia e Insumos Estratégicos. Departamento de Assistência Farmacêutica e Insumos Estratégicos. Assistência farmacêutica na atenção básica: instruções técnicas para sua organização. Brasília: Ministério Público, 2006.

Ministério da Saúde. Portaria GM n. 3.916, 30 de outubro de 1998. Aprova a Política Nacional de Medicamentos. Diário Oficial [da] República Federativa do Brasil, Poder Executivo, Brasília, DF, 10 nov. 1998b. s. 1, n. 215-E, p. 18.

COSTA, L. F. G. Listagem de medicamentos e a decisão proferida na STA N.59-STJ: a justiciabilidade dos direitos sociais. Revista de Direito Sanitário, São Paulo, v. 5, n. 1, p. 119-135, 2004.

DALLARI, D. A. Regulação de medicamentos. Revista de Direito Sanitário, São Paulo, v. 7, n. 1-3, p. 111-116, 2006.

DRUGDEX Drug Evaluations. Pimecrolimus. Thomson Micromedex Healthcare Series, 2007.

FLORIANÓPOLIS. Secretaria Municipal de Saúde. Plano Municipal de Assistência Farmacêutica: 2006-2008. Florianópolis, 2006.

LEITE S. N., MAFRA A. C. Que direito? Trajetórias e percepções de usuários no processo de acesso a medicamentos por mandados judiciais no estado de Santa Catarina. Ciência \& Saúde Coletiva on-line. Disponível em: $<$ www.cienciaesaudecoletiva.com.br>.

LUIZA, V. L.; BERMUDEZ, J. A. Z. Acesso a medicamentos: conceitos e polêmicas. In: BERMUDEZ, J. A. Z.; OLIVEIRA, M. A. Esher A. Acesso a medicamentos: derecho fundamental. Papel del Estado. Rio de Janeiro: ENSP, 2004. p. $45-67$.

MARQUES, S. B. A garantia do direito social à assistência farmacêutica: o papel do sistema jurídico e do sistema político. Revista de Direito Sanitário, São Paulo, v. 7, n. 1-3, p. 195-217, 2006.

MESSEDER N. M.; CASTRO DE, C. G. S. O.; LUIZA, V. L. Mandados judiciais como ferramentas para garantia do acesso a medicamentos no setor público: a experiência do Estado do Rio de Janeiro. Cadernos de Saúde Pública, São Paulo, v. 21, n. 2, p. 525-534, 2005.

RODRIGUES, M. A. P.; FACCHINI, L. A.; LIMA, L. S. Modificações no padrão de consumo de psicofármacos em localidade do sul do Brasil. Revista de Saúde Pública, São Paulo, v. 40, n. 1, p. 107-114, 2006.

SUPREMO TRIBUNAL FEDERAL - STF. Disponível em: <http:// www.stf.gov.br>. 

Nascimento Jr./Benedito Carlos Cordeiro/ Ana Paula Veber

TRAVASSOS, C.; VIACAVA, F.; FERNANDES, C.; ALMEIDA, C. M. Desigualdades geográficas e sociais na utilização de serviços de saúde no Brasil. Ciência \& Saúde Coletiva, São Paulo, v. 5, n. 1, p. 133-149, 2000.

VIEIRA, F. S.; ZUCCHI, P. Distorções causadas pelas ações judiciais à política de medicamentos no Brasil. Revista de Saúde Pública, São Paulo, v. 41, n. 2, p. 214-222, 2007.

WANNMACHER, L. Uso racional de medicamentos: medida facilitadora do acesso. In: BERMUDEZ, J. A. Z.; OLIVEIRA, M. A. Esher A. Acesso a medicamentos: derecho fundamental. Papel del Estado. Rio de Janeiro: ENSP, 2004. p. 91-101.

WHO COLLABORATING Centre for Drugs Statistics Methodology. Anatomical Therapeutic Chemical Classification (ATC code). Disponível em: <http:// www.whocc.no/atcddd>. Acesso em: 15 mar. 2007.

\section{AGRADECIMENTOS}

Programa de Integração Pós-graduação/Graduação UNIVALI; CNPq (Conselho Nacional de Desenvolvimento Científico e Tecnológico). 